\title{
EL CONSTRUCTO CULTURAL DEL BORDE MARÍTIMO- PORTUARIO DE SANTURTZI (BIZKAIA): PAISAJE DE SENSACIONES, EFECTOS Y CREACIONES RELACIONALES
}

\author{
Juan Antonio Rubio-Ardanaz \\ Univ. Extremadura., Dpto. de Psicología y Antropología
}

\section{Resumen}

El borde marítimo-portuario de Santurtzi (Bizkaia) se muestra como realidad viva y dinámica, escenario de sensaciones, efectos y creaciones relacionales. Estos dan lugar a un paisaje, observable etnográficamente, cuyos retazos permiten una visión de espacios y lugares cambiantes y en pleno desarrollo. Al respecto, comprobamos intervenciones públicas y privadas que comportan una nueva configuración de este punto de la costa vasca, radicalmente transformado. En este escenario va ocupando su lugar una fisionomía sociocultural donde destaca la simbiosis entre la pesca, nuevas actividades de carácter cultural y el turismo local. Por otra parte, aparecen llamativas intervenciones urbanísticas (bloques de viviendas, carril de bicicletas, paseo sobre el borde marítimo-portuario) que responden a un significativo aumento de la densidad poblacional y de la actividad industrial. Simbólicamente, la proximidad a la costa sirve de base para suavizar, disfrazar y amortiguar la intensa urbanización en curso, así como los efectos sobre el medio marítimo y costero, incisiva e intensamente industrializado.

Palabras clave: BORDE MARÍTIMO-COSTERO; PAISAJE PORTUARIO; SIMBOLIZACIÓN; URBANIZACIÓN; INDUSTRIALIZACIÓN; SANTURTZI (BIZKAIA)

\section{THE CULTURAL CONSTRUCT OF THE MARITIME-PORT BORDER OF SANTURTZI (BIZKAIA): LANDSCAPE OF SENSATIONS, EFFECTS AND RELATIONAL CREATIONS}

\begin{abstract}
The maritime-port border of Santurtzi (Bizkaia) is shown as a living and dynamic reality, as a scene of sensations, effects and relational creations. These give rise to a landscape, ethnographically observable, whose fragments allow a vision of changing spaces and places in full development. We verify public and private interventions that involve a new configuration of this radically transformed point of the Basque coast. In this scenario, a sociocultural physionomy is taking its place where the symbiosis between fishing, new cultural activities and local tourism stands out. In addition, there are striking urban interventions (housing blocks, bicycle lane, promenade along the sea-port border) that respond to a significant increase in population density and industrial activity. Symbolically, the proximity to the coast serves as a basis to soften, disguise and cushion the intense urbanization underway, as well as the effects on the maritime and coastal environment, incisively and intensely industrialized.
\end{abstract}

Keywords: MARITIME-COASTAL BORDER; PORT LANDSCAPE; SYMBOLIZATION; URBANIZATION; INDUSTRIALIZATION; SANTURTZI (BISCAY)

\footnotetext{
Rubio-Ardanaz, Juan Antonio. 2020. “El constructo cultural del borde marítimo-portuario de Santurtzi (Bizkaia): Paisaje de sensaciones, efectos y creaciones relacionales". AusArt 8 (2): 225-236. DOI: 10.1387/ausart.22002
}

\section{AUSART}




\section{ESPACIO PORTUARIO: PROCESOS, SISTEMAS DE RELACIONES Y ACTIVIDADES SOCIALES}

La interacción con los bordes marítimos, ha ido propiciando escenarios modelados por la acción humana, en virtud de necesidades y objetivos precisos. Colectivos y grupos sociales, cada vez más diversificados, han reconfigurado los espacios de costa y litoral, dejando improntas sobre el territorio que nos hablan del tipo de relaciones en vigor a la hora de llevar a cabo dicha labor. Este queda marcado socioculturalmente por un conjunto de expresiones materiales y simbólicas. En definitiva, la ocupación y la intervención continua y paulatina sobre los lugares, junto o próximos a la mar, ha propiciado una diversidad de situaciones, en función de usos y actividades puestas en marcha a la hora de obtener los recursos materiales pertinentes. Estos parámetros han llevado al estudio de distintas realidades encuadradas bajo el concepto de 'cultura marítima' (Alegret 2006; Carbonell 2010), objeto de estudio disciplinar donde la antropología ocupa un lugar destacable.

En este sentido, a lo largo de la costa vasca, encontramos ciudades y municipios portuarios que comportan paisajes denotativos de una transformación geográfico-productiva. Sobre ellos se han instaurado "mecanismos simbólicos de identificación paisajística" que serán clave para comprender la funcionalidad de sistemas de producción organizados por ejemplo, en torno a la navegación, la logística, o el comercio y la industria entre otros (Vivas \& Lekerikabeaskoa 2018). En esta perspectiva, un acercamiento sobre el terreno, servirá para ir descubriendo un conjunto de manifestaciones comprensivas de las condiciones de vida de los habitantes o lugareños. Y también para perfilar los rasgos de su presencia urbano-portuaria, comprender las desigualdades sociales y territoriales y las disfuncionalidades (o las funcionalidades) comunicativas, tendentes a ciertas formas de aislamiento o privacidad. El espacio portuario, en definitiva, comprende y es un conjunto de procesos, sistemas de relaciones y actividades sociales (Florido 2010).

Esta visión se asocia con un intento por comprender las condiciones espacio-sociales, existentes en pueblos y localidades marítimas. $Y$ al mismo tiempo, saber cómo y desde qué intereses y posiciones se construye y configura el 'confort y la expresión de la personalidad de los espacios'., en nuestro caso urbano-portuarios o marítimos. Perspectiva además vinculada a la de 'medio ambiente', en la que confluyen las actitudes, afectos y roles (propios de vecinos, usuarios, ciudadanos, habitantes, consumidores, agentes sociales y actores). Nos situamos ante dicho marco coincidente con un "urbanismo sen- 
sorial y cronotópico" (Paquot 2009) interactivo y en el que por nuestra parte, no podemos dejar de lado el tipo de relaciones sociales puestas en pie, o en vigor, que están en su base. En este movimiento vivo, dialéctico aparece la controversia entre los espacios públicos y los privados. Los primeros, compartidos y abiertos. Los segundos, limitados, cerrados. $Y$ tanto unos como otros, vigilados y preceptivamente regulados. En ambos casos y a pesar del compromiso ciudadano (gestionado por medio y desde sus correspondientes estrategias políticas), no exentos de tensiones, desigualdades e incluso situaciones violentas.

\section{INCURSIÓN ETNOLÓGICA}

Nuestra incursión analítica se realiza en la localidad de Santurtzi, localidad vizcaína sobre el borde del mar Cantábrico. Municipio culturalmente, tocado por un ambiente de marcada maritimidad, donde inciden la presencia del puerto pesquero y las instalaciones del Puerto de Bilbao. Metodológicamente, planteamos un acercamiento de carácter etnológico a partir de un ejercicio de observación directa, gracias al cual hemos captado los datos que nos han permitido realizar la correspondiente interpretación de la realidad observada. De esta forma, hemos podido conseguir elementos de la novedosa configuración paisajística que está teniendo lugar actualmente de manera sistemática, tratando así de comprender qué encierra en su interior. No pretendemos por lo tanto, 'con-templar' el paisaje marítimo-portuario en sí. Ni tampoco alcanzar el sentimiento del equilibrio funcional, 'con-formativo' y en su caso conducente a una visión 'com-placiente' ante el resultado sociocultural y con aquellos y aquellas que construyen la cultura local.

Aunque el paisaje marítimo-portuario muestra 'formas y manifestaciones', conformando la place, o 'emplazamientos' pertinentes, en un escenario que entendemos compuesto de 'complacencias' (y propuestas y sentimientos sobre los lugares), estas en definitiva, son una oferta para un 'constructo sociocultural', propia de un 'tiempo para el progreso' industrial. No se trata de observar tan solo directamente, quedándonos en los exteriores, sino de encontrar una puerta de acceso al sentido del engranaje profundo que se configura en el momento actual, donde destacan acciones de instancias públicas (consistoriales, gubernamentales, etcétera) y privadas (constructoras, promo- 
toras, etcétera), a lo largo del límite existente en el borde marítimo-portuario del municipio.

Entendemos en todo caso nuestra observación, primero, como un ejercicio primordialmente etnográfico; pero en segundo lugar, conducente a una interpretación de carácter y efecto 'trans-textual'. En este sentido el texto antropológico elaborado aquí, supone la experiencia conducente a un impacto ideacional, y a una toma de conciencia con los que comprender críticamente las realidades. Acontecimientos revestidos y recubiertos de 'progreso postmoderno', pero cuya lógica conduce al sinsentido y contradicciones (un tanto acuciantes) ante un espacio profundamente vaciado y vuelto a rellenar de manera desproporcionada, insostenible y poco respetuosa con el medio ambiente.

\section{SIMBIOSIS DE USOS: PESQUERO, TURÍSTICO Y SIMBÓLICO}

Justo partiendo del puerto pesquero, espacio accesible públicamente, comienza a dibujarse la línea divisoria que contorna el Puerto de Bilbao, separándolo físicamente del resto del municipio. A partir de ella se configuran dos espacios diferenciados, uno de acceso restringido y reservado a las personas que desempeñan sus oficios, labores y profesiones en el recinto portuario industrial, y otro de gestión municipal y de acceso abierto que incluye también el puerto pesquero. Sin embargo, a pesar de esta supuesta separación, visualmente las instalaciones industriales hacen parte de un paisaje propio y habitual, observable desde puntos precisos de dicha línea divisoria. Sobre este borde, aparentemente inerte, limitado a una función fronteriza principalmente, se muestra un dinamismo y movimiento dignos de interés.

La apertura del puerto pesquero se manifiesta actualmente a través de un ejercicio simbiótico en el cual, muelles, rampas y edificios principalmente, han ido abriéndose a actividades netamente diferenciadas de las ejercidas por los pescadores. Una primera prueba de este movimiento, parte del interés del consistorio por dotar al puerto de un nuevo carácter. Desde el ayuntamiento se pone en valor como lugar visitable y apto para un turismo, en un principio de carácter local, promoviendo trabajos de reacondicionamiento entre los que destaca la restauración del viejo edificio de la cofradía (construido en 1916), 
tenido emblemáticamente como principal elemento representativo de la personalidad arrantzale o pescadora de Santurtzi.

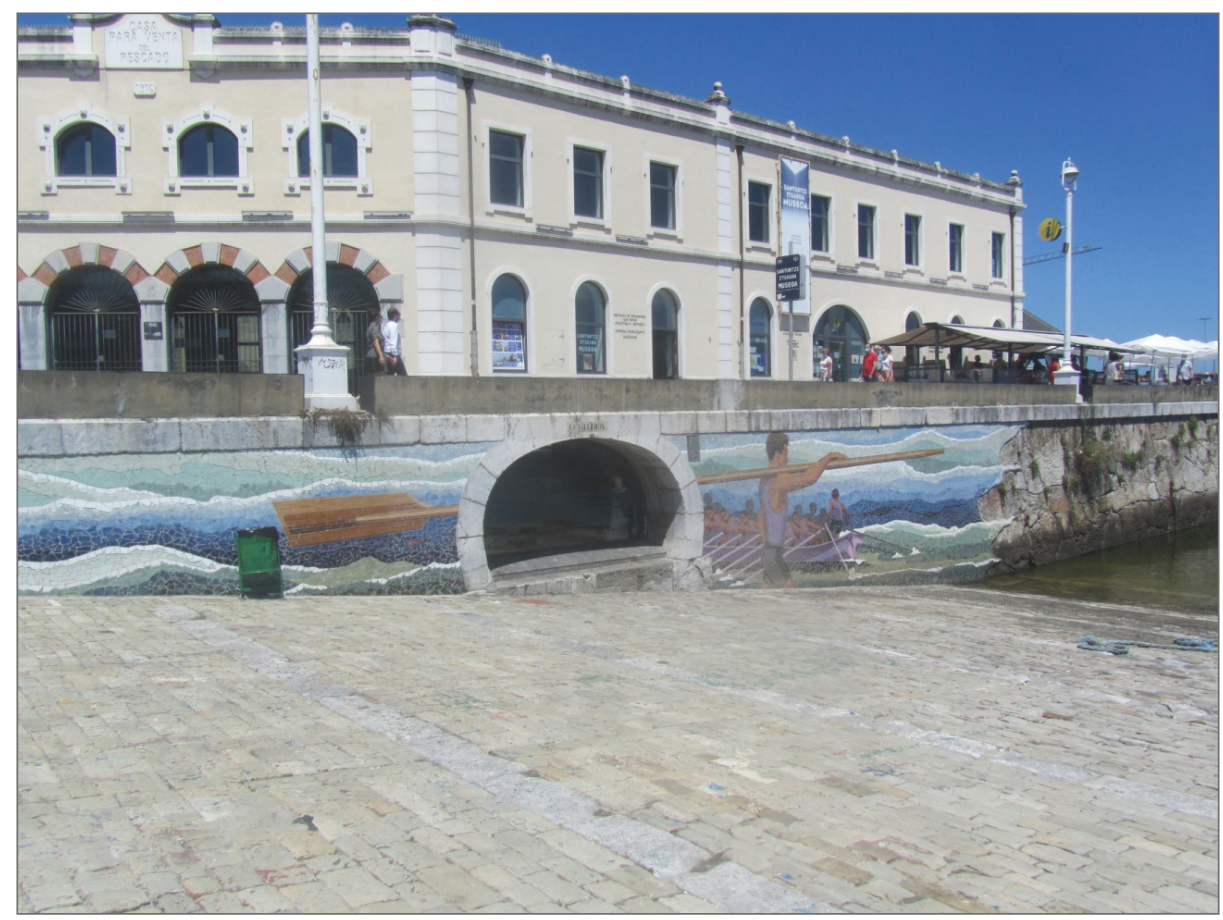

Figura 1. Mosaico homenaje al Club de Remo y edificio de la Cofradía. En la fachada principal los arcos de la lonja de venta pública de pescado y en el primer tramo de la fachada lateral la entrada al centro de interpretación y a la oficina de turismo municipales (2020).

Los pescadores se ven obligados a negociar y velar por sus intereses ante una inminente renovación del edificio, imprescindible y cuyo deterioro con el paso de los años no han sido capaces de solventar. A cambio de la iniciativa, promoción y gestión económica de fondos públicos desde el ayuntamiento en un alto porcentaje procedentes de dicha institución-, este abre una oficina de turismo y el Centro de Interpretación Santurtzi Itsasoa, en los bajos del edificio. A modo de pequeño museo destaca una exposición de carácter histórico, bien considerada y valorada entre los hombres y mujeres pescadores. La renovación se inaugura en 2012, y además de la reforma y rehabilitación general que afecta a otras dependencias (cafetería, el antiguo hogar del pescador, cámara, etcétera), la Cofradía de Pescadores San Pedro de Santurtzi obtiene una oficina renovada y la apertura de una lonja para la venta pública de pescado (figura 1). Estamos ante un juego de intereses y de cambio que comporta nuevas acciones en el puerto, aunque ya anteriormente, cercanos a los puntos 
de amarre de los pesqueros, se han instalado pantalanes para embarcaciones de recreo, aportando una ambientación desconocida hasta el momento.

Este esfuerzo por dotar de nuevas funciones al Puerto pesquero, años más tarde adquiere un matiz interesante, ahora de cariz estético. Consiste en la instalación de sendos mosaicos artísticos (trencadis). Uno representa un grupo de rederas sobre una de las escalerillas laterales del puerto, y otro de mayores dimensiones, una trainera con su tripulación y con un remero como figura principal. Estamos ante una intervención de carácter simbólico-marítimo, aportando un vivo y llamativo colorido sobre sendas paredes de uno de los muelles y de la rampa principal del puerto. Este último, paradójicamente no cobra como referencia la esfera arrantzale, sino el mundo del remo deportivo, profundamente arraigado en Santurtzi. Actualmente representado por el Club de Remo Itsasoko Ama Sotera, fundado en 1967. Su denominación conlleva dos alusiones, por una parte Itsasoko Ama, en honor a la Virgen del Mar, patrona del barrio de Mamariga, en su origen pescador. Y por otra, Sotera, nombre oficial de su trainera, en recuerdo a Sotera San Martín Ortiz (18681938), afamada sardinera.

La creación artística a cargo de Jon Mao Luengo (Barakaldo, 1978), inaugurada en 2015, responde a un homenaje expreso al club remero, atendido desde instancias municipales. $Y$ se instala cercano también a su sede deportiva ubicada en el recinto portuario. Como vemos, cada intervención se acompaña de una carga simbólica, en este último caso expresada materialmente y aportando matices para esta nueva fisionomía portuaria. Usos novedosos vienen a dar este carácter simbiótico al lugar, originariamente arrantzale.

\section{BORDE SOBRE EL ESPACIO URBANO Y EL PORTUARIO-INDUSTRIAL}

Paralelamente, por el primer tramo del borde marítimo-portuario que traza el límite entre el espacio municipal y el Puerto de Bilbao, en su parte más próxima al puerto pesquero, arranca una calle, cuyo nombre es significativo: Itsasalde kalea. Corresponde a un espacio intensa y netamente urbanizado, pero que nos pone sobre aviso de la presencia cercana de la mar. La nomenclatura municipal se ve acompañada por otros apelativos como por ejemplo, el que ostenta el bar Kai Alde Berria que nos indica la cercanía al puerto. Como 
vemos, el espacio urbano, objeto también de simbolización, adquiere una personalidad idealiza por medio de nombres alegóricos con los que vecinos y vecinas recuerdan la personalidad portuaria y marítima del municipio.

No obstante, se constata un contexto de sensaciones contrapuestas. Por un lado ante la gran explanada, prodigada y ocupada por el puerto industrial, oculta tras los edificios que se alzan sobre Itsasalde kalea (y de cuyo desarrollo han sido testigos algunos vecinos y vecinas). Situación esta, habitual para los lugareños, bien visible desde las mismas viviendas. $Y$ también desde dos pequeños puntos abiertos entre los bloques de pisos y lonjas. Estos nos ofrecen la oportunidad de contemplar una primera panorámica -desde la calle- de un paisaje intensamente industrializado. $Y$ aunque en Santurtzi no es difícil seguir la pista del paisaje portuario, podemos asomarnos por estos dos resquicios urbanos que a modo de mirador muestran la otra parte del borde. 'Salir a la calle a hacer la compra y los recaos', a pasear; 'bajar a comprar el pan o bajar el perro', potear -en resumen, socializar en, o a través del espacio público-, encuentra un marco habitual que contrasta con aquel reservado y restringido, propio del Puerto de Bilbao, cuyo borde en esta parte interior se ve acompañado de una vía férrea (figura 2). Estos 'miradores urbano-industriales' nos ofrecen el paisaje de un puerto puntero y altamente activo e industrializado -ofreciendo un importante número de puestos de trabajo-, con leve rumor es apreciable de noche y cuyos efectos contaminantes pasan más bien desapercibidos Contraste en definitiva entre dos espacios objeto de delimitación, que no de separación, sobre el borde marítimo-portuario (figuras 2 y 3 ).

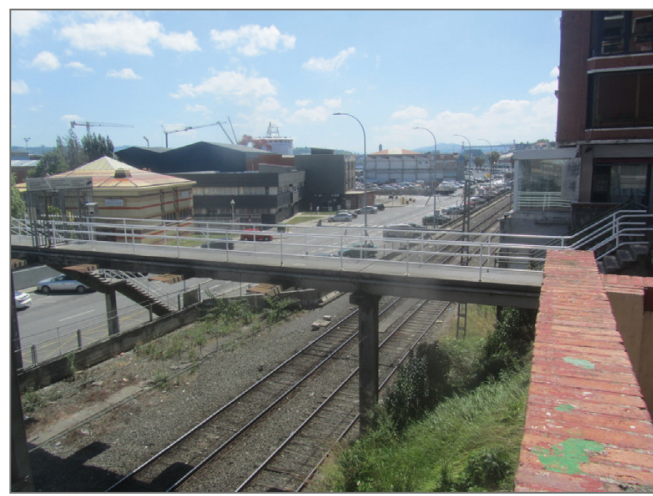

Figura 2. Vista del Puerto de Bilbao desde Itsasalde kalea (núm. 18), y pasarela de acceso restringido a sus instalaciones (2019), (contrastar con la figura 3).

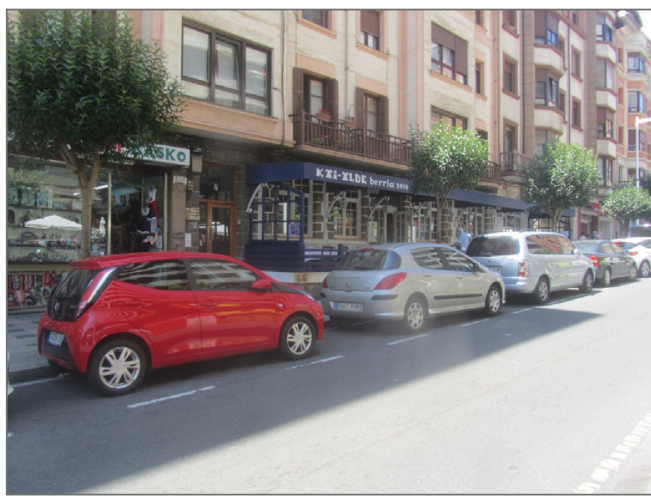

Figura 3. Aspecto y ambiente urbano en Itsasalde kalea sobre el borde limitador del Puerto de Bilbao (2020), (contrastar con la figura 2). 
Bastaría ahora con proseguir hacia el final de la calle, para topar con el Paseo de la Sardinera, donde se abre un amplio y auténtico mirador al puerto, en comparación con los anteriores. En este punto comprobamos un ejercicio y dinámica de simbolización semejantes. Al principio del paseo se encuentra el Café Bar Miramar que despliega una pequeña terraza con sus correspondientes sillas y mesas a disposición de sus clientes y clientas. Lugar habitual para 'tomar un café, leer el periódico o pasar un rato tranquila' (aunque, como nos remarca una clienta habitual, menos frecuentado debido a la pandemia causada por el coronavirus Covid-19, coincidente en parte con nuestras observaciones sobre el terreno). De nuevo se remarca la proximidad a la mar, pero paradójicamente 'mirar y ver el mar' se ha convertido en 'mirar y ver el movimiento y todo el montaje del puerto'. Espacio y emplazamiento poblado por grúas, camiones, contenedores de vivo colorido e instalaciones propias para la estiba y demás labores portuarias.

Muy próximo a este café-bar tiene su sede en una reducida lonja, la Iglesia Evangélica Arrantzaleak (adscrita a la Federación de Entidades Religiosas Evangélicas de España). Un ejercicio de 'trans-fusión' con la cultura tradicional de raíces pescadoras santurtziarra. Un pequeño espejismo nominativo, en un desierto simbólico-religioso, cuya imagen no coincide con una realidad local según la cual no hay ningún pescador de la cofradía que pertenezca a esta 'asamblea' o congregación. Al pretendido carácter arrantzale le acompaña una oferta de culto, tres días a la semana (martes, viernes y domingos). Y contraviniendo el mensaje evangélico contenido en la expresión "dejad que los niños se acerquen a mí", ("no se lo impidáis, pues de los que son como ellos es el reino de Dios", Evangelio de Marcos, capítulo 10, versículos 13-16), comprobamos un expreso cartel a la entrada, que reza "Prohibido jugar al balón. Propiedad privada". Mensaje de esta comunidad cristiana, en un rincón aparentemente público, que transmite un valor y modelo relacional, basado en la 'privacidad' de la propiedad.

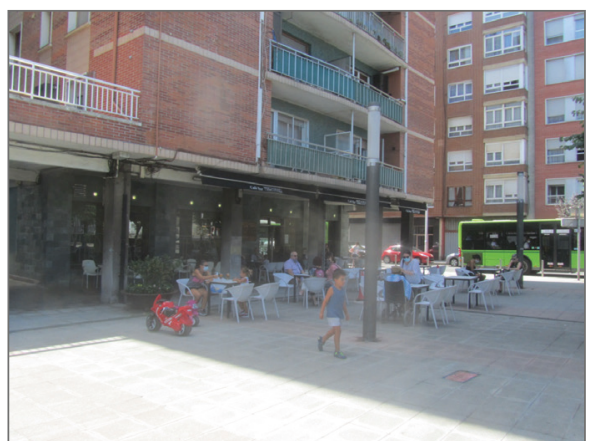

Figura 4. Terraza del Café-Bar Miramar al inicio del Paseo de la Sardinera (2020)
El Paseo de la Sardinera comporta un significativo contenido simbólico y patrimonial (Rubio-Ardanaz 2014). En él se encuentra el "Monumento a la Sardinera - Sardinerari Eskainitako monumentua", escultura de Joaquín Lucarini (1964) ante la cual se desarrolla una parte de los actos conmemorativos del Sardinera Eguna (Homobono 2016), festividad de 
reciente creación -2014- celebrada anualmente en memoria de estas mujeres, vendedoras de pescado en calles y espacios públicos. Y frente al paseo, también encontramos las viviendas del denominado Poblado de Pescadores (1969), en sus inicios propiedad de la cofradía y posteriormente de los pescadores beneficiarios en un principio (pisos en su mayoría vendidos, con el paso del tiempo, a personas ajenas a la comunidad pescadora). Construido a las afueras del núcleo urbano del pueblo y desde el que se divisaba limpiamente la mar (Rubio Ardanaz 2020). Sin embargo, esta zona del municipio será enseguida objeto de nuevas intervenciones urbanísticas, dando lugar en la actualidad a un nuevo ensanche sobre cuya fisionomía, sensaciones y paisaje vamos a detenernos a continuación.

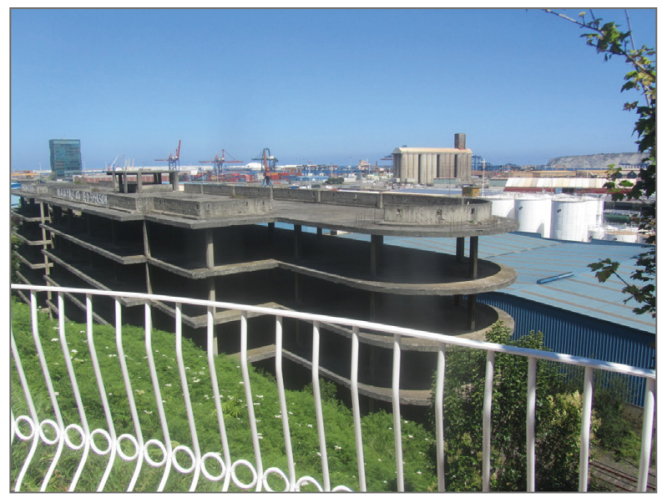

Figura 5. Vista del Puerto de Bilbao desde el Paseo de la Sardinera (2020).

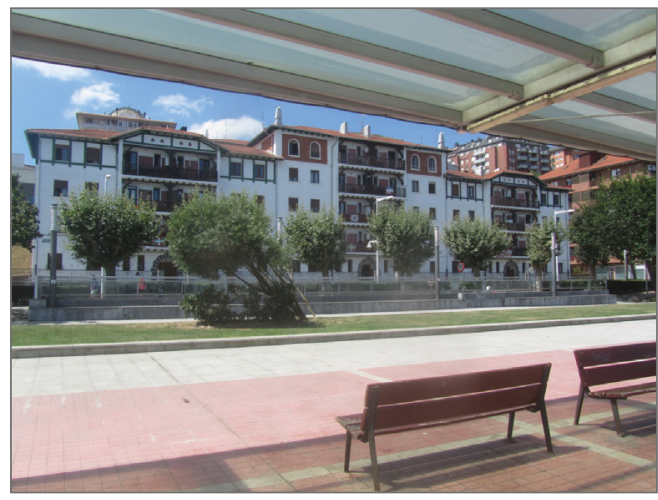

Figura 6. Poblado de los Pescadores frente al Paseo de la Sardinera (2020)

\section{FISIONOMÍA; SENSACIONES Y PAISAJE PARA UN NUEVO ENSANCHE}

Siguiendo el borde nuevamente y en dirección hacia Zierbena ('localidad trans-mutada' sin remisión por los efectos del 'desarrollo' portuario-industrial, donde apenas se mantienen vestigios de su originaria personalidad marítimo-pesquera), una nueva avenida, Iparragirre etorbidea se confunde todavía con la carretera N-639. Esta parte del borde marítimo-portuario ofrece dos aspectos remarcables de reciente intervención. Por una vorágine actividad constructora que va poniendo en pie nuevos bloques de viviendas. Y por otra, de forma orquestada, suavizando el borde limitador, junto a trazos de muro y tramos de vallas de vocación separadora, un nuevo paseo peatonal al que acompaña paralelamente un radiante y recién estrenado carril para bicicletas o bidegorri. 


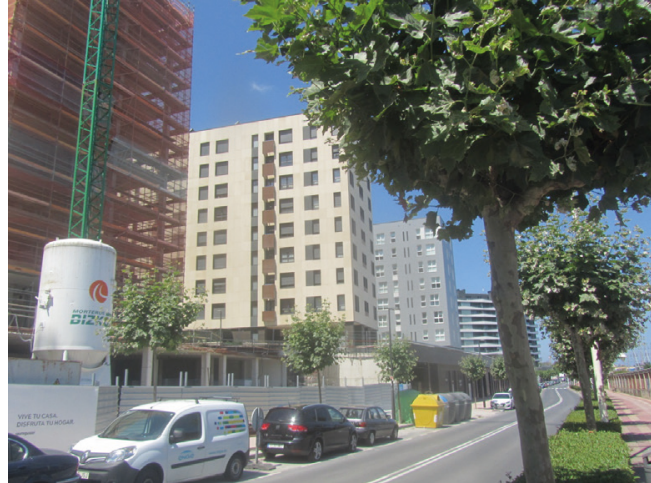

Figura 7. Bloques en construcción con anunciadas "espléndidas vistas al mar" sobre el borde marítimo-portuario (Iparragirre etorbidea), (2020)

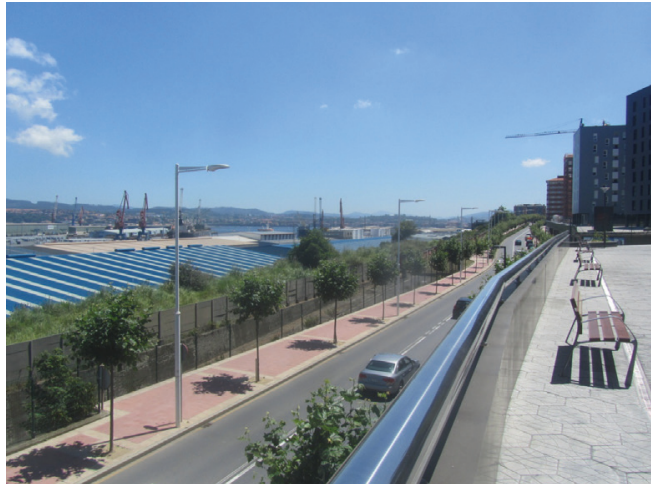

Figura 8. Paisaje portuario, desde el bloque de viviendas en Iparragirre etorbidea (números $95-\mathrm{A}, 95-\mathrm{B}$ y $95-\mathrm{C}$ )

(2020)

Aquí las promotoras promulgan sus propios versículos invitando, desde las tapias que separan todavía las zona en obras: "Vive tu casa, Disfruta tu hogar". Es así como la empresa constructora Jaureguizar propone un lugar donde poder establecer su residencia a los pobladores del municipio. Vivir y disfrutar se convierten en valores adscritos a estos nuevos y abigarrados bloques de pisos ya a la venta y que se están ocupando poco a poco. Todo ello con la oferta de financiación de una entidad bancaria, CaixaBank, la cual anuncia que se trata de "Casas llenas de vida". Y en este capítulo de la promoción, ahora sobre los locales o lonjas a la venta, Jaureguizar, advierte al viandante de que "No es solo un local. Es tu futuro negocio" (indicando el teléfono al que llamar en caso de estar interesado). Viviendas en parte promocionadas en modo de cooperativa: Rompeolas 2020, S. Coop, "a un paso del centro y con vistas al mar". Rompeolas imaginado, malecón ficticio, ante un paisaje en el que apenas es posible distinguir algún trazo de agua marina, entre los extensos terrenos ganados a la mar por el Puerto de Bilbao.

Esta misma dinámica se vuelve a repetir un poco más adelante, en este nuevo 'ensanche' de Santurtzi, ahora sobre los antiguos terrenos de la Compañía Logística de Hidrocarburos, CLH, previamente descontaminados y donde se concentrará un denso número de nuevos pobladores. Como anuncia la empresa promotora, "núcleo urbano privilegiado" con "espléndidas vistas al mar", que en realidad se traducen en 'portuarias e industriales vistas a la no-mar'. Y donde toma cuerpo la tendencia a la privatización, valor en pie sobre los terrenos, ahora recalificados que van haciendo posible esta realidad constructora (Figura 8). El paisaje del borde marítimo portuario sobre el que se alzan estos nuevos bloques, no obstante se verá suavizado, con un paseo 
poblado artificiosamente de arbolitos perfectamente alineados y un reluciente bidegorri.

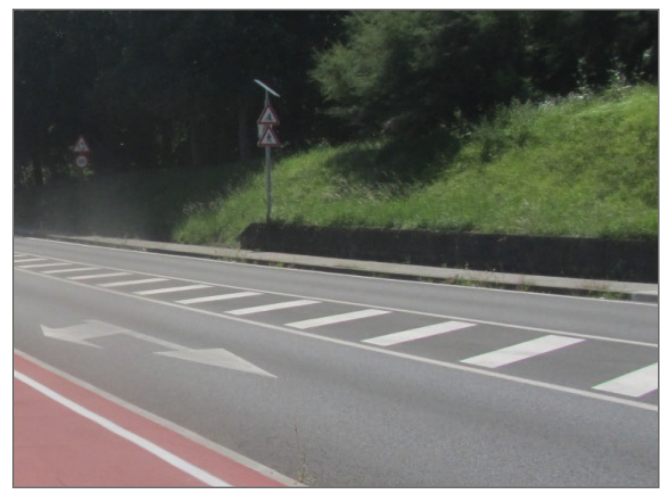

Para cerrar el recorrido realizado hasta aquí, podemos concluir que recorrer etnográficamente el borde marítimo-costero, nos ha permitido verificar un conjunto de manifestaciones socioculturales, entendiéndolo como lugar dinámico, objeto de transformaciones precisas, correlacionadas directamente, por una parte con la proximidad y presencia a un activo puerto industrial de progreso continuo. Y por otra de una expansión urbana que no es ajena a la proximidad marítima. No se trata de un límite inerte y simplemente separador de dos realidades (portuario-industrial y urbano-vecinal). El proceso urbanístico en marcha que conlleva nuevas edificaciones, infraestructuras y servicios, como hemos podido confirmar, se ve acompañado de la 'construcción cultural' de una nueva vecindad que responde a un modelo de identidad y confort que pasan por una habitabilidad sobre el borde marítimo-portuario, con una puesta en valor que ofrece paisajes y vistas realmente paradójicos.

\section{Referencias bibliográficas}

Alegret i Tejero, Joan Lluís. 2006. Etnologia de les societats marítimes: Perspectives d'anàlisi, àmbits d'estudi i propostes de treball. Palamós: Universitat de Girona, Càtedra d'Estudis Marítims Museu de la Pesca

Carbonell i Camós, Eliseu. 2010. "La patrimonialización de un paisaje marítimo: de la arena de la playa a la arena política". Pasos, Revista de Turismo y Patrimonio Cultural 8(4): 569-81

Florido del Corral, David. 2010. El espacio portuario de Conil: Infraestructuras socioeconómicas, políticas y de ingeniería. Sevilla: Agencia Pública de Puertos de Andalucía

Homobono Martínez, José Ignacio. 2016. Fiestas marítimas populares: Patrimonio inmaterial de la Ría de Bilbao, El Abra y la costa occidental de Bizkaia. Prólogo de Isusko Vivas Ziarrusta; presentación de Jon Ruigómez Matxin Bilbao: Museo Marítimo Ría de Bilbao

Paquot, Thierry. 2009. L'espace public. Paris: La Découverte 
Rubio Ardanaz, Juan Antonio. 2014. Antropología y maritimidad: Entramados y constructos patrimoniales en el Abra y Ría de Bilbao. Bilbao: Museo Marítimo Ría de Bilbao

- . 2020. "Las cofradías de pescadores en el País Vasco: Cambios e influencias históricas en la pesca de bajura; El caso de Santurtzi (Bizkaia)". Estudios Atacameños 64: 39-65

Vivas Ziarrusta, Isusko \& Amaia Lekerikabeaskoa Gaztañaga. 2018. "Espacios marítimo-portuarios en la Ría de Bilbao: Desde su extensión metropolitana hasta la Ribera de Deusto y la península-isla de Zorrotzaurre". En La Ribera de Deusto y Zorrotzaurre en la Ría de Bilbao: Análisis urbano, sociocultural y estético del espacio marítimo, Isusko Vivas Ziarrusta, Isusko \& Amaia Lekerikabeaskoa Gaztañaga, dirs., 211-42. Bilbao: Museo Marítimo Ría de Bilbao

(Artículo recibido: 13-09-20; aceptado: 27-11-20) 